\title{
IMPACTO DE LOS MÉTODOS DE ENSEÑANZA DE LA LECTURA EN LA COMPRENSIÓN LECTORA
}

\section{IMPACT OF READING TEACHING METHODS ON READING COMPREHENSION}

\author{
Antonio Motos Valero ${ }^{1}$ \\ Universidad Oberta de Catalunya (UOC) \\ amotosv@uoc.edu \\ Beatriz Martín del Campo² \\ bmartind@uoc.edu \\ Universidad de Castilla La Mancha (UCLM)
}

\begin{abstract}
Resumen
Existe una importante controversia sobre la idoneidad de los diferentes métodos de enseñanza de la lectura para obtener una buena comprensión lectora. El presente estudio fue diseñado para conocer el impacto que tienen los métodos de lectura iniciados en Educación Infantil en la comprensión lectora en Educación Primaria. Con este fin, se seleccionó una muestra de 54 alumnos

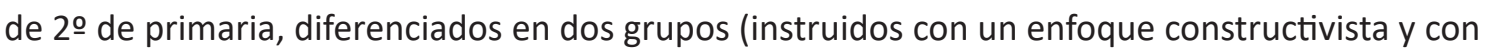
un método sintético-fonético). En primer lugar, se realizó un análisis cualitativo con entrevistas y un diario de campo a partir de la observación en las aulas. En segundo lugar, se pasó la prueba PROLEC-R a ambos grupos, comparando sus puntuaciones en velocidad, precisión y comprensión lectora. No se encontraron diferencias significativas en cuanto a la velocidad, comprensión lectora y precisión de lectura de palabras. En cambio, en la lectura de pseudopalabras, los resultados obtenidos por el alumnado del método sintético-fonético fueron significativamente superiores. Estos resultados sugieren la necesidad de buscar métodos alternativos que tengan presentes los beneficios que conlleva el aprendizaje del código escrito, pero sin dejar de lado la construcción del significado desde una perspectiva práctica, funcional y significativa considerando la lectura y la escritura como prácticas sociales.

Palabras clave: Alfabetización - Lectura - Métodos de lectura - Comprensión lectora - Aprendizaje lector
\end{abstract}




\begin{abstract}
There is an important controversy about the suitability of the different reading teaching methods to obtain a quality reading comprehension. For this reason, the present study was designed to know the impact that reading methods started in Early Childhood Education have on reading comprehension in Primary Education. With this purpose, a sample of 542 nd grade students was selected, differentiated into two groups (instructed with a constructivist approach and with a synthetic-phonetic method). First, a qualitative analysis was carried out through interviews and a field diary. Secondly, the PROLEC-R test was carried out on both groups, analysing their results based on speed, precision and reading comprehension. No significant differences were found in terms of speed, reading comprehension and word reading precision. On the other hand, in the reading of pseudowords, the results obtained were significantly higher by the syntheticphonetic method students. These results suggest the need to search for alternative methods that take into account the benefits of learning the written code, but without neglecting the construction of meaning from a practical, functional and meaningful perspective, changing the vision of literacy and turning it into a social practice.
\end{abstract}

Key Words: Literacy - Reading - Reading methods - Reading comprehension - Reading acquisition 


\section{INTRODUCCIÓN}

La lectura es uno de los pilares fundamentales en la formación de la sociedad actual y, por tanto, se debe enseñar con el método más adecuado para que su adquisición sea de calidad, potenciando, no solo la descodificación de las palabras, sino también una comprensión lectora adecuada a cada edad. En el actual sistema educativo español, la comprensión lectora sigue siendo uno de los puntos débiles, haciéndose patente, en informes internacionales como PIRLS y PISA, las importantes dificultades que presentan los estudiantes en la comprensión de textos (Llamazares, Alonso y Sánchez, 2015). Por ello, es de crucial importancia indagar qué método es el más adecuado para mejorar la comprensión lectora de los escolares.

Existen dos posturas enfrentadas en cuanto al aprendizaje de la lectura. Por un lado, encontramos la necesidad del aprendizaje del código escrito. En este sentido, autores como Fons (2008), entienden este aprendizaje de la lectura como una intervención de procesos de bajo nivel, como descodificar o reconocer palabras, con otros de alto nivel como son la interpretación de datos, la formulación de hipótesis o la anticipación y discusión de la información relevante. Además, otros autores como Adams (2001), añaden los beneficios que conlleva para el alumnado con dificultades, dada sus características de aprendizaje, una enseñanza explícita y sistemática de la fonética, ayudándoles a asimilar el código escrito y, por tanto, a reconocer las palabras de manera rápida, fácil y eficaz.

En la otra parte de esta confrontación, encontramos otros autores (Fuentes, Calderin y Pérez, 2017; Pascual, Madrid y Estrada, 2018), que entienden el aprendizaje de la lectura como un proceso en el que el lector debe darle sentido al texto $y$, al mismo tiempo, relacionarlo con sus conocimientos previos, acción que le permite conocer y comprender su entorno. En este sentido, el proceso de aprendizaje de la lectura se entiende como algo que va más allá de una relación y transformación grafema-fonema, defendiendo que este aprendizaje debe ser funcional y darse en contextos sociales y significativos (Benveniste, 2002). Asimismo, siguiendo a Martín-del-Campo (2011), para el aprendizaje de la lectura por parte del alumnado con dificultades, no será suficiente con entrenar la capacidad para transcribir el lenguaje oral a lo escrito, sino que será necesario tener en cuenta los contextos sociales para crear situaciones significativas y funcionales.

En este sentido, el Modelo Simple de Lectura (Gough y Tunmer, 1986; Hoover y Gough, 1990) plantea que la comprensión lectora se basa en dos componentes: el componente de decodificación y reconocimiento de palabras y el componente de comprensión oral. Gough (1996) contrapone su modelo R=DxLC a los planteamientos del Whole Language o Lenguaje Integrado (Goodman, 1969; Goodman, 1986; Martín-del-Campo, 1996), según los cuales el aprendizaje de la lectura es un proceso natural de adivinación psicolingüística que no precisa de una instrucción explícita de la estructura fonológica de las palabras. 
A partir de entonces, muchas investigaciones dirigieron sus esfuerzos a demostrar que la instrucción de la lectura debía ser sistemática y centrada en la decodificación (Ehri et al., 2001. Ver Bowers, 2020, que pone en cuestión que esta enseñanza tenga que ser sistemática) aunque, como señala Share (2008), el anglocentrismo reinante en esta investigación no nos ofrece una visión adecuada sobre el aprendizaje del reconocimiento de palabras en lenguas transparentes, como el español. La visión simple de lectura de Gough y Tunmer (1986) se situó en un punto intermedio.

Sin embargo, existe una importante brecha entre la práctica educativa actual y la evidencia científica sobre la comprensión lectora y su enseñanza. El desconocimiento de los docentes encargados del proceso de enseñanza de la lectura se hace patente y existe una clara falta de información y conocimiento práctico sobre las nuevas evidencias científicas (Fuentes, Calderín y Pérez, 2017), corroborando que, a pesar de haber recibido una capacitación permanente, la práctica educativa no se ve mejorada (Fuentes et. al., 2017). De acuerdo con estos autores, las creencias y percepciones sobre el aprendizaje de la lectura que tienen los docentes se asocian en ocasiones al desconocimiento conceptual y otras a sus prejuicios.

No obstante, la alfabetización escolar despierta el interés y la preocupación de un amplio porcentaje de docentes e investigadores, dado que cuando un estudiante no alcanza la plena alfabetización en las etapas iniciales de su escolarización, sus posibilidades de adquirir conocimientos sobre contenidos de otras áreas se reducen notablemente (De Mello y Porta, 2017). Esta idea, también es defendida por Elzbieta (2014), al afirmar que el proceso de aprendizaje de la lectura será determinante para que el aprendiz tenga éxito durante su proceso educativo, ya que cualquier déficit que surja en este proceso tendrá serias repercusiones en su formación. Asimismo, otros autores como González et al. (2018) ponen énfasis en que la intervención del proceso de enseñanza-aprendizaje de la lectura, para que aporte beneficios y se disminuyan las dificultades en el aprendizaje escolar debe darse en etapas tempranas.

Pero lo fundamental es ser capaces de detectar el proceso de enseñanza y aprendizaje mediante el cual el niño y la niña conectan, captan e interactúan con el texto (Villar y Vieiro, 2015). Por tanto, no podemos fijarnos en el resultado del aprendizaje de la lectura si no estudiamos con detalle la manera a la que accede el niño/a a la lectura.

Al seguir con el estudio realizado por Villar y Vieiro (2015), podemos destacar que existen dos vías principales para llevar a cabo el proceso de enseñanza-aprendizaje de la lectura: la que transforma los signos gráficos en sonidos y la que conecta de manera directa los signos gráficos con el significado. En la actualidad, se desarrolla una variedad de métodos importante, pero como afirman Villar y Vieiro, los métodos sintéticos, los 
métodos globales, así como la perspectiva constructivista, siguen siendo los métodos más utilizados como vía de acceso a la lectura.

En este estudio, nos centraremos en dos de ellos: en el método sintético-fonético y el enfoque constructivista. Por un lado, los métodos sintéticos-fonéticos, siguiendo la descripción realizada por Villar y Vieiro (2015), son métodos que priorizan el desciframiento de letras y fonemas para alcanzar una lectura más precisa y rápida, sin dar importancia al contexto y la comprensión lectora. Además, como añaden De Mello y Porta (2017), inicialmente se enseñan los fonemas y grafemas, luego, las sílabas, palabras y frases, comenzando por las vocales y, progresivamente, las consonantes las cuales se asocian a un objeto y/o personaje. De esta forma, se permite la fácil y rápida identificación de cualquier tipo de palabra, tanto las que son conocidas y frecuentes para el niño/a como las que son completamente desconocidas para ellos/as o son menos frecuentes en su contexto.

Por otra parte, la perspectiva constructivista, según De Mello y Porta (2017), tiene como finalidad fomentar la curiosidad del alumnado para que aprenda de manera natural, utilizando el contexto como recurso de aprendizaje. Los alumnos/as son guiados por el docente para que sean capaces de encontrar las respuestas a partir de sus propios conocimientos, de su interacción con la realidad y con sus iguales y relacionando los nuevos aprendizajes con los que ya posee.

Actualmente, aunque se utilizan diversas metodologías en las aulas para potenciar los procesos de lectura y escritura, muchos estudiantes presentan dificultades para comprender los textos y, en consecuencia, su rendimiento escolar y su pensamiento creativo se ve perjudicado (Rivas, 2015). Por esta razón, este autor relaciona la comprensión lectora con el desarrollo educativo del niño/a ya que, cuando el estudiante comprende lo que está leyendo, amplía exponencialmente su bagaje cultural y adquiere gran cantidad de información y, por tanto, de conocimiento.

Existen autores que destacan que el fin último de la lectura es comprender el texto (Hjetland et al., 2019; Tighe, Wagner y Schatschneider, 2015). Además, otros estudios recientes indican que la comprensión lectora es la habilidad de la lectura más importante, destacando la fase de descodificación en la lectura como un momento crítico para el desarrollo de este proceso (Gidalew y Van den Berg, 2018; Wang et al., 2019). Asimismo, otros autores definen la comprensión lectora como la capacidad de emplear el texto para el aprendizaje y relacionarlo con las ideas que ya se poseen, siendo una habilidad fundamental y determinada por la comprensión del lenguaje y de su decodificación (Hjetland, et. al., 2019; Rivas, 2015; Stanley, Petscher y Catts, 2018). Abusamra et al., (2020) muestran, en una investigación con adolescentes entre 13 y 15 años, que las variables de bajo nivel que mejor explican la comprensión lectora son el vocabulario, 
la lectura de no palabras y la inhibición verbal. Esto nos podría llevar a pensar que las habilidades de decodificación están detrás de la competencia en habilidades lectoras incluso en edades avanzadas y que el uso de metodologías de enseñanza de la lectura que trabajen de manera explícita el componente fonológico son necesarias para un adecuado desarrollo de la comprensión lectora.

Sin embargo, aunque podemos encontrar algunas investigaciones que relacionan diversos métodos de enseñanza y su impacto en el rendimiento de la lectura (Ghasemi y Vaez-Dalili, 2019; Hjetland et al., 2019; Rivas, 2015; Robinson, 2018), estas son escasas o poco relevantes. De esta manera, el interés y novedad de la presente investigación radica en la relación que se establece entre el proceso inicial de aprendizaje de la lectura y su impacto en las habilidades de comprensión lectora del alumnado. Por tanto, se relaciona, de manera directa, el método utilizado para la enseñanza de la lectura en sus etapas iniciales (Educación Infantil) con la compresión lectora en etapas posteriores (Educación Primaria).

En este contexto, el estudio que aquí se presenta pretende investigar el impacto que tienen dos de los métodos más utilizados en la iniciación de la lectura en la etapa de Educación Infantil, como son el método sintético-fonético y el enfoque constructivista, en la comprensión lectora del alumnado de Educación Primaria. Por tanto, como punto de partida para la presente investigación, debemos reflexionar y preguntarnos lo siguiente: ¿qué método de enseñanza de la lectura tiene un impacto más positivo en la comprensión lectora del alumnado, el método sintético-fonético o el enfoque constructivista? En relación con la pregunta planteada, se espera que, con el método sintético-fonético, los alumnos/as puedan leer antes al tener la llave para descifrar los códigos escritos y convertirlos en fonemas, aumentando así la velocidad de lectura, pero al segmentarla, esto pueda tener consecuencias más negativas para la comprensión lectora. Por otro lado, se espera que el enfoque constructivista, aunque el proceso de aprendizaje de decodificación y reconocimiento de palabras es más lento, la comprensión lectora en etapas posteriores sea mayor, al centrarse en la enseñanza de construcción del significado del texto.

\section{Método}

\section{Participantes}

La muestra del estudio estuvo formada por 54 sujetos de 20 de Educación Primaria de dos centros educativos de la provincia de Alicante. Estos fueron divididos en dos grupos, en función del método de lectura al que estuvieron expuestos desde su etapa de Educación Infantil (enfoque constructivista o sintético-fonético). 
El primer grupo (en adelante, Grupo 1), formado por 27 sujetos, utiliza como método de enseñanza de la lectura un enfoque constructivista. La media de edad es de 7 años y 5 meses (rango de edad comprendido entre 7 años y 1 mes y los 8 años y 0 meses). Las maestras que tuvieron durante la etapa de infantil y durante los dos primeros cursos de primaria también forman parte de la muestra del estudio.

El segundo grupo (en adelante, Grupo 2), formado por 27 sujetos, utiliza como método de lectura el método sintético-fonético desde la etapa de Educación Infantil. La media de edad es de 7 años y 6 meses (rango de edad comprendido entre 7 años y 2 meses y los 8 años y 0 meses). También forman parte de la muestra la maestra de infantil y la maestra de primaria encargadas del proceso lector de este grupo.

De los 54 sujetos de la muestra, $42,59 \%$ eran niñas y $57,41 \%$ niños. Dentro de la muestra encontramos una alumna extranjera, pero fue escolarizada desde los 3 años en el centro escolar y no presenta ninguna dificultad con el idioma. Además, ha sido excluido de la muestra el alumnado con necesidades educativas especiales.

Los padres/madres y, en su defecto, los tutores legales de los menores objeto de estudio firmaron un consentimiento informado antes del inicio del estudio. Además, los diferentes docentes implicados firmaron una licencia de cesión de derechos de imagen, voz y derechos de explotación, puesto que las entrevistas que se realizaron fueron grabadas en audio. Asimismo, el protocolo de la investigación se adhiere a los principios éticos del centro donde se realiza el estudio, así como a los principios generales que regentan la ética de la investigación como la Declaración de Helsinki (Asociación Médica Mundial, 2013) y lo establecido en el Reglamento General de Protección de Datos (Reglamento 2016/679, 2016).

\section{Instrumentos}

Para la realización del estudio se emplearon diferentes instrumentos cuantitativos como el PROLEC-R (Cuetos et al., 2014) y el BADyG E1 (Yuste y Yuste, 2011), e instrumentos cualitativos como un diario de campo en forma de registro observacional del entorno y una entrevista semiestructurada creada ad hoc para el estudio (ver Figura 1) siguiendo los principios establecidos por Fábregues et al. (2016).

Figura 1.

Entrevista semiestructurada

Etapa educativa:

Educación Infantil $\square \quad$ Educación Primaria

Método de enseñanza de la lectura:

Sintético-fonético $\square \quad$ Enfoque constructivista 


\section{Guion de preguntas:}

1. ¿Qué importancia tiene para usted la lectura en el aula? ¿Por qué?

2. ¿Cuál es el proceso que siguió para que los alumnos/as aprendieran a leer?

3. ¿En qué orden se introdujeron las letras?

4. ¿Cómo se relacionaba cada letra con su fonema?

5. ¿Cómo era una sesión tipo dedicada al área de lectura?

6. ¿Qué material utilizó para leer con los alumnos/as?

7. ¿Cuánto tiempo dedicó a leer con los niños/as?

8. ¿Leían grupalmente en el aula?

9. ¿Cómo propiciaba el gusto por la lectura?

10. ¿Trabajó la comprensión lectora con los alumnos/as? ¿Cómo lo hizo?

11. ¿Cuál era el objetivo final que buscaba con la aplicación de ese método de lectura?

Por un lado, con el PROLEC-R se realizó una evaluación de los procesos que intervienen en la comprensión escrita y detección de dificultades en la capacidad lectora. De este modo, se evaluó la identificación de letras, el reconocimiento de palabras, los procesos sintácticos y los procesos semánticos, a través de nueve tareas como son: nombre o sonido de las letras, igual-diferente, lectura de palabras, lectura de pseudopalabras, estructuras gramaticales, signos de puntuación, comprensión de oraciones, comprensión de textos y comprensión oral (Cuetos, et al., 2014). Con el uso del PROLEC-R, además de evaluar, en general, el desarrollo lector de los sujetos, se evaluó la comprensión lectora de los estudiantes, actuando esta como variable dependiente. Asimismo, al considerar los tiempos de ejecución junto con los aciertos para determinar la precisión y eficiencia lectoras, se controló la aparición de variables extrañas como la posible falta de fluidez lectora en los sujetos.

Por otra parte, con el BADyG E1 se evaluaron las aptitudes diferenciales y generales de los sujetos a través de algunas pruebas básicas: relaciones analógicas, problemas numéricoverbales, matrices, cálculo numérico, órdenes verbales complejas y figuras giradas (Yuste y Yuste, 2011). Una vez pasado el BADyG E1 se obtuvieron los cocientes intelectuales (CI) de los estudiantes, controlando así que no existan diferencias significativas que puedan afectar a los resultados.

Y, por último, antes y durante la recogida de datos, se pasó una entrevista semiestructurada a las maestras que los sujetos de la investigación tuvieron en Educación Infantil, para conocer en detalle la metodología que se llevó a cabo durante esta etapa, así como a las maestras de primaria para conocer los detalles del método aplicado durante el curso 
anterior, durante 10 de primaria. Para la elaboración de la entrevista se partió de un guión donde se recogieron las ideas relevantes de las que se quería obtener información y se plantearon una serie de preguntas abiertas, ofreciendo así a las entrevistadas una mayor libertad de respuesta y con mayores posibilidades de expresión (Fábregues, et al., 2016). Asimismo, el primer autor entró en cada una de las aulas en dos sesiones (una de 50 minutos en el grupo 1 y una de 30 en el grupo 2), en las que se elaboró un diario de campo mediante la observación directa y un registro descriptivo del entorno, referido al método de enseñanza de la lectura que se lleva a cabo durante el curso del estudio (2은 de primaria).

Todo esto ayudó a describir con detalle los métodos de lectura, permitiendo comprender, de manera global, el proceso de aprendizaje de la lectura por el que habían pasado los niños/as desde 3 años de infantil hasta 20 de primaria. Asimismo, con esta información se controló el grado de estandarización de los métodos de enseñanza de la lectura utilizados, actuando estos como variable independiente del estudio (De Mello y Porta, 2017; Maturana y Garzón, 2015).

\section{Procedimiento}

La investigación llevada a cabo es mixta y de tipo transversal y retrospectiva, ya que se tiene en cuenta el método de aprendizaje de la lectura desde su inicio en la etapa de infantil hasta $2 \circ$ de primaria, pero sin realizar un estudio longitudinal. En el diseño de la investigación se plantearon las siguientes variables: la comprensión lectora y la velocidad de lectura como variables dependientes y el método de enseñanza-aprendizaje de la lectura (sintético-fonético o enfoque constructivista) como variable independiente: se compararon dos grupos que habían sido instruidos en Educación Infantil con estos dos métodos. Además, fueron controladas otras variables intervinientes como la edad, el sexo y el $\mathrm{Cl}$.

En primer lugar, antes de realizar la investigación, y para controlar otras variables extrañas que pudieran influir en la comprensión lectora de los sujetos como la falta de vocabulario o la falta de conocimiento sobre el contenido del texto o sobre su contexto, se instruyó al profesorado de primaria del Grupo 1 y Grupo 2 para que ofrecieran, previamente, información sobre los textos y significados relevantes que se evaluaron en la prueba PROLEC-R.

Posteriormente, se realizaron las entrevistas a las maestras de infantil y primaria y se elaboró el diario de campo en las aulas de 20 de primaria. Ambos procedimientos se llevaron a cabo, paralelamente, durante el desarrollo del estudio. Para la entrevista se contactó a las dos maestras de infantil y las dos de primaria, y se concretó una cita para realizarla individualmente y en sus respectivas aulas, para así poder observar también el 
material utilizado y la ambientación de las mismas. Para cada una de las entrevistas se necesitó un tiempo aproximado de 30-45 minutos. Una vez finalizadas las entrevistas, las cuales fueron grabadas en audio, se realizó una transcripción de las mismas. Con las dos maestras de primaria, además de realizar la entrevista para obtener información sobre cómo se aplicó el método de lectura en 10 de primaria, se realizó un diario de campo, en el que se registró la metodología que se estaba usando en 2ำ de primaria, y así poder obtener una descripción global de todo el proceso. La observación en el aula se llevó a cabo en una sesión de 45 minutos en cada uno de los dos grupos-clase.

Paralelamente a las entrevistas y el diario de campo, se pasaron los diferentes instrumentos cuantitativos del estudio, durante las primeras semanas de mes de enero. Antes de realizar las pruebas, se les explicó a los niños/as en que iban a consistir y se resolvieron todas las dudas. Por un lado, el PROLEC-R se realizó de manera individual y se necesitó una sesión de entre 20-30 minutos por cada sujeto, realizándose esta prueba en una sala de reuniones donde se evitaron diversas distracciones. Se disponía de dos horas diarias para pasar el PROLEC-R, necesitando, por tanto, un total de entre 9 y 13 días lectivos para poder completar la prueba con toda la muestra. Por otra parte, para el BADyG E1 se necesitaron dos sesiones grupales de 45 minutos aproximadamente para cada grupo. Las dos sesiones se hicieron en días consecutivos y durante la segunda semana de enero.

Finalizado el estudio cuantitativo, se recogieron los resultados y contabilizaron los datos. Para el PROLEC-R se atendió al número de aciertos junto con los tiempos de reacción y en el BADyG E1 se tuvieron en cuenta el número de aciertos, errores y omisiones.

\section{Análisis estadístico}

La corrección, baremación y realización de informes del BADyG E1 se realizó mediante la aplicación online, mientras que el procedimiento del análisis del PROLEC-R fue de manera manual. De ambos instrumentos cuantitativos se obtuvieron como unidad de medida puntuaciones numéricas, clasificadas y ordenadas en una escala de Razón o Proporción.

Una vez recogida la muestra de datos cuantitativos, se llevaron a cabo dos técnicas a través del software estadístico SPSS. Por una parte, se realizó un análisis univariante de las variables intervinientes del estudio (Cociente Intelectual y edad) obteniendo la media aritmética del Grupo 1 y el Grupo 2. Por otra parte, se elaboró un análisis bivariante, analizando los resultados a través de un contraste entre las medias obtenidas, entre el Grupo 1 y el Grupo 2, en los valores de las variables dependientes (velocidad y comprensión lectora). Para comprobar si las diferencias entre las distintas puntuaciones de los dos grupos de estudio eran significativas, se aplicó la prueba t de Student y se 
estableció el valor de probabilidad en el $5 \%(p=0,05)$, suponiendo que para valores menores de 0,05 las diferencias sí eran significativas.

Para las entrevistas y el diario de campo se realizó, con el procesador de textos Word, un análisis cualitativo de contenido para el cual se siguieron las siguientes fases: en primer lugar, se realizó una transcripción de las entrevistas y un registro descriptivo de la observación directa; posteriormente, se leyeron y analizaron las respuestas organizándolas en diferentes códigos, subtemas y temas (lectura, proceso de enseñanza, literatura y comprensión lectora); y finalmente, se elaboró un análisis de frecuencias de los códigos obtenidos y se analizó las diferencias entre ambos métodos con el test Chi cuadrado a través de SPSS.

\section{Resultados}

Con la finalidad de dar respuesta a la pregunta del presente estudio sobre qué método de enseñanza de la lectura (enfoque constructivista o sintético-fonético) tiene un impacto más positivo sobre la comprensión, en primer lugar, se realiza una descripción sobre algunas variables intervinientes de la muestra.

En el Grupo 1, 66,67\% eran niños y 33,33\% eran niñas; y en el Grupo 2, 48,15\% eran niños y $51,85 \%$ eran niñas. Además, de la prueba BADyG se obtuvo que la media del $\mathrm{Cl}$ del Grupo 1 ( $M=97,33$; DT=17,202) es superior a la del Grupo $2(M=96,44 ; D T=11,396)$. Pero como se observa en la Tabla 1, los resultados de la Prueba t de Student demuestran que la diferencia de $\mathrm{Cl}$ entre ambos grupos no es significativa.

\section{Tabla 1.}

Prueba t de Student sobre Cl

\begin{tabular}{|c|l|c|c|c|c|}
\cline { 3 - 6 } \multicolumn{2}{c|}{} & \multicolumn{4}{c|}{ prueba t para la igualdad de medias } \\
\cline { 3 - 6 } \multicolumn{2}{c|}{} & $\mathrm{t}$ & $\mathrm{gl}$ & $\begin{array}{c}\text { Sig. } \\
\text { (bilateral }\end{array}$ & $\begin{array}{c}\text { Diferencia } \\
\text { de medias }\end{array}$ \\
\hline $\mathrm{Cl}$ & $\begin{array}{l}\text { Se asumen varianzas } \\
\text { iguales }\end{array}$ & 0,224 & 52 & 0,824 & 0,889 \\
\hline
\end{tabular}

Una vez descrita la muestra, procedemos a informar sobre el análisis de las entrevistas y el diario de campo, con el fin de presentar con claridad las diferencias entre los métodos de enseñanza de la lectura a los que estaban sometidos los participantes. En la Tabla 2 se muestra el sistema de categorías generado en un proceso inductivo de AC (análisis de contenido) (Schreier, 2012) de los cuadernos de campo y la transcripción de las entrevistas semiestructuradas. 
Tabla 2.

Sistema de categorías

\begin{tabular}{|c|c|}
\hline Subtemas & Códigos \\
\hline $\begin{array}{l}\text { Prerrequisitos de la } \\
\text { lectura }\end{array}$ & $\begin{array}{l}\text { Ritmo de aprendizaje y madurez } \\
\text { Requisitos para adquisición de la lectura }\end{array}$ \\
\hline $\begin{array}{l}\text { Decodificación y } \\
\text { aprendizaje }\end{array}$ & $\begin{array}{l}\text { Presencia de las letras en el aula } \\
\text { Lectura por parte del adulto } \\
\text { Tipo de enseñanza } \\
\text { Metodología de enseñanza de la lectura } \\
\text { Aprendizaje de las grafías } \\
\text { Aprendizaje de los fonemas } \\
\text { Formación de sílabas uniendo el fonema con las vocales }\end{array}$ \\
\hline Aprendizaje y refuerzo & $\begin{array}{l}\text { Propuestas relacionadas con lectura } \\
\text { Actividades para el aprendizaje de la grafia y sonido } \\
\text { Priorización del aprendizaje práctico } \\
\text { Explicación teórica } \\
\text { Refuerzo lectura alumnos con dificultades } \\
\text { Refuerzo de la lectura y contenidos en casa }\end{array}$ \\
\hline Papel del alumno & Papel activo y protagonista del alumno \\
\hline Papel del adulto & $\begin{array}{l}\text { Papel de guía del adulto } \\
\text { Papel activo del adulto }\end{array}$ \\
\hline Agrupación de trabajo & $\begin{array}{l}\text { Grupos cooperativos } \\
\text { Trabajo individual }\end{array}$ \\
\hline $\begin{array}{l}\text { Importancia de la } \\
\text { lectura }\end{array}$ & $\begin{array}{l}\text { Valoración de la lectura en el aula } \\
\text { Ejemplo lector por parte del adulto }\end{array}$ \\
\hline Tiempos de lectura & $\begin{array}{l}\text { Ausencia de hora fija de lectura } \\
\text { Lectura a demanda } \\
\text { Temporalización flexible } \\
\text { Frecuencia de lectura por parte del adulto } \\
\text { Frecuencia lectura del alumno } \\
\text { Refuerzo lectura en otros ámbitos }\end{array}$ \\
\hline Lectura significativa & $\begin{array}{l}\text { Necesidad de relación entre escuela y sociedad exterior } \\
\text { Aprendizaje funcional, motivacional y significativo } \\
\text { Variedad de carteles escritos por toda el aula } \\
\text { Relación con otras materias }\end{array}$ \\
\hline Agrupación lectora & $\begin{array}{l}\text { No lectura grupal } \\
\text { Lectura individual } \\
\text { Lectura grupal }\end{array}$ \\
\hline $\begin{array}{l}\text { Normativa sobre } \\
\text { lectura }\end{array}$ & $\begin{array}{l}\text { Objetivos de la etapa de infantil referidos al proceso de } \\
\text { lectura/escritura }\end{array}$ \\
\hline Enlace curriculum & Juegos para el aprendizaje de los contenidos gramaticales \\
\hline Tipos de texto & $\begin{array}{l}\text { Variedad de tipos de textos y tipologías } \\
\text { Aprendizaje sobre la elaboración de diferentes textos } \\
\text { Letra ligada no presente en el aula } \\
\text { Libro de lectura individual } \\
\text { Texto asociado a los contenidos teóricos explicados }\end{array}$ \\
\hline Género literarios & $\begin{array}{l}\text { Variedad materiales de lectura en el aula } \\
\text { Género narrativo el más utilizado }\end{array}$ \\
\hline Biblioteca de aula & $\begin{array}{l}\text { Biblioteca con variedad de géneros literarios y tipologías } \\
\text { Biblioteca pequeña con poco protagonismo y poca variedad }\end{array}$ \\
\hline $\begin{array}{l}\text { Comprensión de } \\
\text { textos }\end{array}$ & $\begin{array}{l}\text { Falta de trabajo de la comprensión por parte de los alumnos } \\
\text { Relación entre lectura y comprensión del texto } \\
\text { Comprensión de frases sencillas } \\
\text { Comprensión de palabras de media frecuencia } \\
\text { Preguntas de comprensión del texto leído }\end{array}$ \\
\hline Comprensión oral & Trabajo de la comprensión oral a partir de la lectura del adulto \\
\hline
\end{tabular}


En la Tabla 3 se muestran los porcentajes de las frecuencias de los códigos en cada grupo. Estos porcentajes se establecen de manera global para cada subtema y son contabilizados según la frecuencia en la que aparecen los diferentes códigos, englobados en cada subtema, tanto en el análisis de las entrevistas como en el diario de campo. Así, por ejemplo, para establecer el porcentaje en ambos métodos en el que se enseñaban prerrequisitos de la lectura, se computaron la frecuencia en el que aparecían en la transcripción de la entrevista y en el diario de campo, varios códigos como: ritmo de aprendizaje y madurez y requisitos para la adquisición de la lectura.

A través del test Chi cuadrado, se confirman que las diferencias entre ambos métodos son significativas, ya que el valor de significancia asintónica bilateral es menor al 5\% $(p=0,001<0.05)$. El valor experimental del estadístico Chi cuadrado es de $42,549 y$, por medio de una tabla de contribuciones, los códigos responsables de la significación entre ambos métodos son: "aprendizaje de grafías, fonemas y sílabas y presencia letras en aula" $(7,13)$, "lectura a demanda, ausencia hora fija lectura y temporalización flexible" $(5,57)$ y "comprensión de palabras y frases" $(4,9)$.

\section{Tabla 3.}

Porcentajes de frecuencia de códigos

\begin{tabular}{|c|c|c|c|}
\hline & & Método de e & señanza \\
\hline & & $\begin{array}{c}\text { Enfoque } \\
\text { constructivista } \\
\end{array}$ & $\begin{array}{l}\text { Sintético- } \\
\text { fonético }\end{array}$ \\
\hline Código & PROCESO DE ENSEÑANZA & $52,5 \%$ & $47,5 \%$ \\
\hline & Prerrequisitos de la lectura & $85,7 \%$ & $14,3 \%$ \\
\hline & Aprendizaje de grafías, fonemas y sílabas y presencia de letras en aula & $16,7 \%$ & $83,3 \%$ \\
\hline & Refuerzo de la lectura & & $100,0 \%$ \\
\hline & Aprendizaje práctico, papel activo alumnado y grupos cooperativos & $100,0 \%$ & \\
\hline & Explicación teórica y trabajo individual & & $100,0 \%$ \\
\hline & LECTURA & $64,4 \%$ & $35,6 \%$ \\
\hline & Lectura a demanda, ausencia hora fija lectura y temporalización flexible & $100,0 \%$ & \\
\hline & Lectura significativa & $58,8 \%$ & $41,2 \%$ \\
\hline & Lectura individual y grupal & $16,7 \%$ & $83,3 \%$ \\
\hline & LITERATURA & $56,3 \%$ & $43,8 \%$ \\
\hline & Variedad tipos de texto y tipologías & $100,0 \%$ & \\
\hline & Biblioteca de aula y variedad de materiales de lectura & $55,6 \%$ & $44,4 \%$ \\
\hline & Lectura individual asociada a contenidos teóricos & & $100,0 \%$ \\
\hline & COMPRENSIÓN LECTORA & $50,0 \%$ & $50,0 \%$ \\
\hline & Comprensión de palabras y frases & & $100,0 \%$ \\
\hline & Comprensión oral & $100,0 \%$ & \\
\hline & Relación entre lectura y comprensión & $75,0 \%$ & $25,0 \%$ \\
\hline
\end{tabular}


A continuación, para dar respuesta a las hipótesis sobre si un método conlleva más beneficios sobre la comprensión lectora que el otro método, analizamos los resultados del PROLEC-R. En cuanto a la velocidad de lectura de palabras y pseudopalabras, como se esperaba, la media en el método sintético-fonético ( $M=69,11$; $D T=17,357$ y $M=93,59$; $D T=22,738)$ es ligeramente superior que en el enfoque constructivista ( $M=67,04$; $D T=18,453$ y $M=91,44 ; D T=24,968)$, pero estas diferencias no son significativas, como se puede ver en la Tabla 4.

Tabla 4.

Prueba t de Student sobre velocidad de lectura

\begin{tabular}{|l|l|c|c|c|c|}
\cline { 3 - 6 } \multicolumn{2}{c|}{} & \multicolumn{4}{|c|}{ prueba t para la igualdad de medias } \\
\cline { 3 - 6 } \multicolumn{2}{c|}{} & $\mathrm{t}$ & $\mathrm{gl}$ & $\begin{array}{c}\text { Sig. } \\
\text { (bilateral }\end{array}$ & $\begin{array}{c}\text { Diferencia } \\
\text { de medias }\end{array}$ \\
\hline $\begin{array}{l}\text { Velocidad Lectura } \\
\text { Palabras }\end{array}$ & $\begin{array}{l}\text { Se asumen } \\
\text { varianzas iguales }\end{array}$ & $-0,425$ & 52 & 0,672 & $-2,074$ \\
\hline $\begin{array}{l}\text { Velocidad Lectura } \\
\text { Pseudopalabras }\end{array}$ & $\begin{array}{l}\text { Se asumen } \\
\text { varianzas iguales }\end{array}$ & $-0,331$ & 52 & 0,742 & $-2,148$ \\
\hline
\end{tabular}

Por otro lado, la media de precisión de lectura de palabras en el método sintético-fonético $(M=39,22 ; D T=0,974)$, aunque sutilmente superior, es prácticamente igual que la media del enfoque constructivista ( $M=38,96 ; D T=1,315)$. Estas diferencias no son significativas. Sin embargo, en cuanto a la precisión de lectura de pseudopalabras, la media en el enfoque constructivista $(M=35,78 ; D T=3,479)$ es inferior a la media del método sintéticofonético $(M=37,52 ; D T=2,502)$. En este caso, la diferencia sí es significativa, como podemos observar en la Tabla 5.

Tabla 5.

Prueba t de Student sobre precisión de lectura

\begin{tabular}{|l|l|c|c|c|c|}
\cline { 3 - 5 } \multicolumn{2}{c|}{} & \multicolumn{4}{c|}{ prueba t para la igualdad de medias } \\
\cline { 3 - 6 } \multicolumn{2}{c|}{} & $\mathrm{t}$ & $\mathrm{gl}$ & $\begin{array}{c}\text { Sig. } \\
\text { (bilateral }\end{array}$ & $\begin{array}{c}\text { Diferencia } \\
\text { de medias }\end{array}$ \\
\hline $\begin{array}{l}\text { Velocidad de } \\
\text { lectura de palabras }\end{array}$ & $\begin{array}{l}\text { Se asumen } \\
\text { varianzas iguales }\end{array}$ & $-0,823$ & 52 & 0,414 & $-0,259$ \\
\hline $\begin{array}{l}\text { Velocidad de lectura } \\
\text { de pseudopalabras }\end{array}$ & $\begin{array}{l}\text { Se asumen } \\
\text { varianzas iguales }\end{array}$ & $-2,111$ & 52 & 0,040 & $-1,741$ \\
\hline
\end{tabular}

En relación con los resultados obtenidos en el PROLEC-R, la media en la comprensión de oraciones en el método sintético $(M=14,81 ; D T=1,545)$ es levemente superior a la media del enfoque constructivista ( $M=14,70 ; D T=2,181)$. Sin embargo, esta diferencia no es significativa. 
Y en cuanto a la media en la comprensión de textos y en la comprensión oral en el enfoque constructivista ( $M=10,41 ; D T=3,478$ y $M=3,59 ; D T=1,151)$ es ligeramente superior a la media del método sintético-fonético ( $M=9,78 ; D T=3,080$ y $M=3,44 ; D T=0,801)$. Pero como podemos ver en la Tabla 6 , estas diferencias tampoco son significativas.

Tabla 6.

Prueba t de Student sobre comprensión lectora

\begin{tabular}{|l|l|c|c|c|c|}
\cline { 3 - 5 } \multicolumn{2}{c|}{} & \multicolumn{4}{c|}{ prueba t para la igualdad de medias } \\
\cline { 3 - 6 } \multicolumn{2}{c|}{} & $\mathrm{t}$ & $\mathrm{gl}$ & $\begin{array}{c}\text { Sig. } \\
\text { (bilateral }\end{array}$ & $\begin{array}{c}\text { Diferencia } \\
\text { de medias }\end{array}$ \\
\hline $\begin{array}{l}\text { Comprensión de } \\
\text { oraciones }\end{array}$ & $\begin{array}{l}\text { Se asumen varianzas } \\
\text { iguales }\end{array}$ & $-0,216$ & 52 & 0,830 & $-0,111$ \\
\hline Comprensión de textos & $\begin{array}{l}\text { Se asumen varianzas } \\
\text { iguales }\end{array}$ & 0,704 & 52 & 0,484 & 0,630 \\
\hline Comprensión oral & $\begin{array}{l}\text { Se asumen varianzas } \\
\text { iguales }\end{array}$ & 0,549 & 52 & 0,586 & 0,148 \\
\hline
\end{tabular}

\section{Discusión}

El propósito de esta investigación ha sido comprobar cuál es el impacto de los dos métodos más utilizados en la iniciación de la lectura en la etapa de Educación Infantil, como el sintético-fonético y el enfoque constructivista, en la comprensión lectora del alumnado en 20 de Educación Primaria. Por ello, al inicio nos preguntamos: ¿qué método de enseñanza de la lectura tiene un impacto más positivo en la comprensión lectora del alumnado, el método sintético-fonético o el enfoque constructivista?

Comenzamos valorando los resultados cualitativos obtenidos. Estos resultados confirman que existen diferencias significativas en cuanto a los métodos de enseñanza a los que han estado expuestos ambos grupos, a partir de un análisis cualitativo de las entrevistas realizadas a las maestras que enseñaron a leer a los sujetos del estudio cuando ambos estaban en educación infantil. En este sentido, la maestra que usó el método sintéticofonético enfoca su enseñanza al aprendizaje sistemático de grafías, fonemas y sílabas, reforzando la lectura individual y grupal y centrándose, principalmente, en enseñar la clave para la conversión grafema-fonema. Por su parte, la maestra que empleaba el enfoque constructivista, se centra en la significatividad de la lectura, utilizando diferentes textos y tipologías textuales más relacionados con el contexto cercano del alumnado. Aún así, hemos de ser cautos a la hora de interpretar los resultados, teniendo en cuenta que la pureza de los métodos no es absoluta y que la experiencia lectora de los niños y niñas también deriva de su entorno no escolar. 
En cuanto a los resultados de las pruebas estandarizadas, la velocidad de lectura tanto de palabras como de pseudopalabras, en contra de lo esperado, no presentan diferencias significativas entre ambos grupos. Sin embargo, sí es significativa la diferencia en la precisión de lectura de pseudopalabras. Por tanto, los resultados confirman que, en el grupo que ha estado expuesto al método sintético-fonético, se desarrollan mecanismos de lectura más precisos, aunque no más veloces, que en el grupo expuesto al enfoque constructivista, aunque estos mecanismos no arrojan diferencias cuando se emplean en palabras reales. Podríamos decir que en los niños que han estado expuestos al método constructivista, las reglas de conversión grafema-fonema no están tan sólidamente adquiridas, aunque eso no afecta a la lectura de palabras y, por tanto, es lógico que no afecte a la comprensión. En la comprensión de oraciones y textos y la comprensión oral, planteamos la hipótesis de que en el enfoque constructivista se daría un mayor nivel de comprensión, ya que utiliza un acercamiento holístico hacia la lectura, teniendo como base la construcción de significados. Sin embargo, los resultados indican que no existen diferencias significativas entre ambos grupos y, por tanto, podemos afirmar que ninguno de los métodos produce ventajas en la comprensión lectora.

En resumen, algunos resultados indican que las hipótesis sobre la relación negativa entre la enseñanza con métodos sintético-fonéticos y la comprensión lectora no se han podido confirmar. Por otra parte, aunque el grupo que aprendió a leer con estos métodos lee con mayor exactitud las pseudopalabras, su comprensión lectora no es mayor que la del grupo "constructivista". Esto es así incluso en la prueba de comprensión de oraciones, en la que el cometer más errores de descifrado puede conducir con más probabilidad a una falta de comprensión, al no disponer de contexto para dar sentido a las palabras desconocidas (Keenan, Betjemann y Olson, 2008).

Por tanto, parece que la comprensión lectora no depende del método que se usa para enseñar a leer y a escribir. Aunque el método sintético-fónico conduce a unas habilidades de descifrado más exactas, la comprensión no se ve afectada. Tampoco existen diferencias en la velocidad de lectura, de modo que los niños y niñas del método constructivista parecen desarrollar fluidez lectora a pesar de una enseñanza no sistemática del código. No obstante, los datos cualitativos nos indican que este aspecto no se descuidaba del todo por parte de la maestra que seguía el método constructivista, por lo que serían necesarias más investigaciones en las que se comparasen metodologías de manera más controlada, una con enseñanza explícita del código y otra sin esta.

Por otra parte, la realización de estudios longitudinales en los que se controle la forma de enseñanza de la lectura a lo largo de los primeros cursos de primaria y su incidencia en la comprensión lectora sería de gran relevancia. De esta forma, se podría comprobar si los efectos de los métodos de enseñanza en la comprensión lectora aparecen más 
adelante, cuando los mecanismos de descifrado del código ya están consolidados (Tobia y Bonifacci, 2015).

Existen pocos estudios relevantes que relacionen los métodos de enseñanza de la lectura de una lengua transparente como el castellano con su repercusión directa en la comprensión lectora. No obstante, hay algunas investigaciones que recogen resultados similares. Cuetos y Suárez-Coalla (2009) tampoco encontraron diferencias en la velocidad de lectura entre grupos de escuelas diferentes y metodologías distintas. Cabe aclarar que estos autores se centran en la precisión de lectura y no en la comprensión, y comparan dos grupos con métodos de enseñanza diferentes, pero con características similares como son un método fonético y uno de combinación fonético-silábico.

Robinson (2018) obtiene resultados coincidentes al afirmar que no existen diferencias significativas en los logros de lectura entre un grupo cuya instrucción se basa en la fonética y entre otro instruido con un método de lenguaje integrado. No obstante, en el estudio de este autor sí existen diferencias significativas en las puntuaciones obtenidas entre el grupo que ha aprendido con un enfoque más constructivista con un tercer grupo con el que se ha puesto en práctica un enfoque basado en fonética, pero más intensivo, donde este último obtiene mejores resultados en los logros de lectura.

Además, Guzmán (1997) confirma con sus resultados que en la lectura de pseudopalabras el alumnado instruido con un método fonético muestra mayores ventajas que el alumnado del método global, ya que estos últimos muestran mayores errores y tiempos de reacción en la lectura de pseudopalabras. Con estos resultados, este autor confirma que el grupo instruido con un método fonético tiene un mayor conocimiento y dominio de las reglas de transformación grafema-fonema.

Sin embargo, al mismo tiempo, Guzmán (1997) no encuentra diferencias significativas en la lectura de palabras no familiares y de larga longitud. Esta diferencia en los resultados puede deberse tanto a la variable de longitud como a la edad de la muestra, ya que estos corresponden al alumnado de primero, en el que puede existir todavía una falta de automatización de las reglas de transformación grafema-fonema. También cabe destacar que Guzmán centra su estudio en analizar las vías de acceso al léxico, la tarea de nombrar y los errores cometidos en relación con palabras de diferente longitud y familiaridad, a diferencia de la prueba PROLEC-R utilizada en el presente estudio que amplía este análisis a la comprensión de frases y textos.

Asimismo, en contra de los resultados obtenidos, en el estudio realizado por Taylor, Davis y Rastle (2017) el grupo centrado en el aprendizaje de sonidos obtiene mejores resultados en la lectura en voz alta y en la comprensión lectora que el grupo instruido con un método basado en el significado. Destacar que este estudio se realiza a través de un paradigma de lenguaje artificial, con adultos y dos lenguas desconocidas para ellos. 
Los hallazgos obtenidos por Villar y Vieiro (2015) indican que los tiempos de lectura en los estudiantes que aprenden a partir del método global son más bajos, siendo estos resultados contrarios a los obtenidos en este estudio. Esto podría deberse a que Villar y Vieiro estudian los tiempos y precisión de lectura de palabras, atendiendo a la longitud, familiaridad y frecuencia silábica posicional, pero no en relación con la lectura de frases y textos más largos, reduciendo la comprensión a palabras sueltas. No obstante, los resultados obtenidos por estos autores coinciden en confirmar que el alumnado del método sintético obtiene mejores resultados en la lectura de palabras de frecuencia léxica baja.

Los resultados encontrados pueden ser de gran utilidad en la mejora de la práctica educativa para reflexionar y eliminar algunas creencias existentes de que la enseñanza de la lectura debe hacerse a través de un método concreto. Esta afirmación se debe a que los resultados sugieren que ambos métodos tienen un efecto semejante sobre la adquisición de la comprensión lectora del alumnado, pero no así con los mecanismos de automatización de descifrado. Por tanto, podemos afirmar que el método sintéticofonético conlleva grandes beneficios para la automatización de la conversión grafemafonema. Además, no debemos olvidar que este aprendizaje explícito del código es necesario para mejorar el aprendizaje lector por parte de los alumnos y alumnas con dislexia, al mejorar las habilidades de decodificación fonológica (Papanicolaou et. al., 2003).

A raíz de los resultados, desde el enfoque constructivista no se debe menospreciar el aprendizaje sistemático y explícito del alfabeto, fonemas y sílabas. Es necesario reflexionar e incorporar los puntos fuertes de uno en la puesta en práctica del otro, siempre teniendo en mente las necesidades y características del alumnado. De acuerdo con Bowers (2020), existe un problema en el enfoque actual. La enseñanza sistemática de la fonética tiene un amplio apoyo dentro de la literatura de investigación, pero no hay evidencias de que un método tenga mejores resultados que otro, por lo que debemos de empezar a considerar métodos alternativos y mixtos para la enseñanza de la lectura.

Es importante abordar el aprendizaje explícito del código escrito y la conversión grafemafonema, pero sin olvidar la necesidad de construir significados y la funcionalidad del lenguaje escrito. Esta enseñanza completa y conjunta es todavía más necesaria en el alumnado con dificultades relacionadas con la lectura, siendo imprescindible una enseñanza del código escrito de manera sistemática y estructurada que le ayude a su adquisición e interiorización, pero reforzando este aprendizaje en contextos sociales significativos. En este sentido, siguiendo a Martín del Campo (2011), es necesario cambiar la visión del uso del lenguaje escrito y convertirla en una práctica social, que haga de la lectura una función práctica y significativa que ayude a su integración social y personal. 


\section{CONCLUSIONES}

Este estudio aporta nuevos conocimientos sobre la importancia de los métodos de enseñanza de la lectura en las etapas iniciales y su relación con la comprensión lectora, siendo este un campo donde las investigaciones son escasas. El carácter del castellano como lengua transparente hace especialmente importante el que existan estudios realizados específicamente en esta lengua.

No obstante, esta investigación tiene algunas limitaciones como una muestra reducida a dos grupos de alumnos/as, por lo que sería interesante replicarlo con una muestra mayor y con características diferentes, incorporando nuevas variables como el contexto sociocultural y económico del alumnado y la motivación y la implicación del profesorado y de las familias. Asimismo, como ya hemos mencionado anteriormente, sería interesante realizar un estudio longitudinal que permita un mayor control de todas las variables intervinientes, e incluir otras medidas relacionadas con la eficacia de los métodos, como la calidad del razonamiento, el pensamiento crítico y la creatividad.

Se espera que los resultados obtenidos con la investigación contribuyan en cierta medida a desdibujar las creencias sociales y de algunos/as maestros/as de la incompatibilidad entre ambos métodos. Quizás no exista la necesidad de elegir entre un método u otro, sino que se deben aprovechar las ventajas que ofrecen cada uno de ellos. El objetivo es mejorar la calidad de la enseñanza de la lectura, siendo conscientes de los beneficios que conlleva el aprendizaje del código escrito, especialmente en el alumnado con dificultades, pero sin dejar de lado la construcción del significado desde una perspectiva práctica, funcional y significativa. Además, con los resultados obtenidos queda de manifiesto la necesidad de reforzar la comprensión lectora, empezando con la comprensión oral y progresando hacia la comprensión de palabras y frases en la etapa de Educación Infantil, no limitando el aprendizaje de la lectura a la descodificación de palabras, sino acompañando el aprendizaje del código escrito del significado de la lengua escrita. En conclusión, con la presente investigación, hemos pretendido abrir un pequeño agujero en el muro existente entre los defensores de métodos como el sintético-fonético y entre los que defienden un enfoque constructivista, haciendo visibles que ambos métodos no son incompatibles, sino que se complementan y ayudan a formar lectores competentes.

\section{REFERENCIAS BIBLIOGRÁFICAS}

Abusamra, V.; Difalcis, M.; Martínez, G.; Low, D. M. \& Formoso, J. (2020). Cognitive skills involved in reading comprehension of adolescents with low educational opportunities. Languages, 5(3), 34. https://doi.org/10.3390/languages5030034

Adams, M.J. (2001). Alphabetic anxiety and explict, systematic phonics instruction: A cognitive science perspective. En S.B. Neuman y D.K. Dickinson (Eds.), Handbook of early literacy research (vol. 1, p.66-88). Guilford Press. 
Asociación Médica Mundial. (2013). Declaración de Helsinki de la AMM: Principios éticos para las investigaciones médicas en seres humanos. 64a Asamblea General. Fortaleza, Brasil. https://www.wma.net/es/policies-post/declaracion-de-helsinkide-la-amm-principios-eticos-para-las-investigaciones-medicas-en-seres-humanos/

Benveniste, C. (2002). La escritura, irreductible a un "código". En E. Ferreiro (Comp.), Relaciones de (in)dependencia entre oralidad y escritura. Gedisa.

Bowers, J.S. (2020). Reconsidering the Evidence That Systematic Phonics Is More Effective Than Alternative Methods of Reading Instruction. Educational Psychology Review 32, 681-705. doi:10.1007/s10648-019-09515-y

Cuetos, F.; Rodríguez, B.; Ruano, E. y Arribas, D. (2014). PROLEC-R. Batería de Evaluación de los Procesos Lectores, Revisada (5a edición). TEA Ediciones.

Cuetos, F. y Suárez-Coalla, P. (2009). From grapheme to Word in Reading acquisition in Spanish. Applied Psycholinguistics, 30, 583-601. doi:10.1017/S0142716409990038

De Mello Francatto, R. y Porta, M. E. (2017). Estrategias Pedagógicas de Alfabetización y su efecto en el Aprendizaje Inicial de la Lecto-Escritura. Revista De Orientación Educacional, 31(60), pp. 22-45. http://www.roe.cl/index.php/roe/article/view/22

Ehri, L.; Nunes, S.; Stahl, S. \& Willows, D. M. (2001). Systematic phonics instruction helps students learn to read: Evidence from the National Reading Panel's meta-analysis. Review of educational research, 71(3), 393-447. https://doi. org/10.3102/00346543071003393

Elzbieta, A. (2014). Invented spelling - a window on early literacy. Edukacja, 5(130), $112-$ 123. https://doaj.org/article/a5909fafc6b04728af5ecb67b5388cbc

Fábregues, S.; Meneses, J.; Rodríguez-Gómez, D. y Paré, M.H. (2016). Técnicas de Investigación Social y Educativa. Editorial UOC.

Fons, M. (2008). Despacito y buena letra. Aula de infantil, 45, 38-41. http://hdl.handle. net/11162/23487

Fuentes, L.; Calderin, N. y Pérez, A. (2017). Creencias y conocimientos de los docentes sobre la enseñanza de la lectura. Revista de estudios interdisciplinarios, 19(2), 343365. http://ojs.urbe.edu/index.php/telos/article/view/161

Ghasemi, B. y Vaez-Dalili, M. (2019). Effect of Three Methods of Morphological Awareness on Iranian Intermediate EFL Learners' Reading Comprehension. International Journal of Instruction, 12(2), 623-638. https://eric.ed.gov/?id=EJ1211010

Gidalew, T. y Van den Berg, G. (2018). The relationship between lecturers' beliefs and their actual methods of reading instruction: An Ethiopian case study. Reading \& Writing 9(1), 162. doi:10.4102/rw.v9i1.162 
González, M.; Martín, I.; Rivas, T. y Prieto, G. (2018). Análisis del rendimiento y de la mejora en la Lectura y en la Escritura en Educación Infantil. Revista de Educación, (382), 225-247. doi: 10.4438/1988-592X-RE-2018-382-398

Goodman, K. (1969). Analysis of Oral Reading Miscues. Applied Psycholinguistics. Reading Research Quarterly, 5, pp 9-30.

Goodman, K. (1986). What's Whole in Whole Language? Heinemann.

Gough, P.B. (1996). How children learn to read and why they fail. Annals of Dyslexia 46, 1-20. https://doi.org/10.1007/BF02648168

Gough, P. B., \& Tunmer, W. E. (1986). Decoding, reading and reading disability. Remedial and Special Education 7, 6-10. doi:10.1177/074193258600700104

Guzmán, R. (1997). Métodos de lectura y acceso al léxico. [Tesis doctoral no publicada]. Universidad de la Laguna, España. https://riull.ull.es/xmlui/handle/915/10070

Hoover, W. A. \& Gough, P. B. (1990). The simple view of reading. Reading and Writing, 2, 127-160. doi:10.1007/BF00401799

Hjetland, H.; Lervåg, A.; Lyster, S.; Hagtvet, B.; Hulme, C. y Melby-Lervåg, M. (2019). Pathways to Reading Comprehension: A Longitudinal Study From 4 to 9 Years of Age. Journal of Educational Psychology, 111(5), 751-763. Doi: 10.1037/edu0000321

Keenan, J. M.; Betjemann, R. S. \& Olson, R. K. (2008). Reading comprehension tests vary in the skills they assess: Differential dependence on decoding and oral comprehension. Scientific Studies of Reading, 12(3), 281-300. https://doi. org/10.1080/10888430802132279

Llamazares, M.; Alonso, M. y Sánchez, S. (2015). Factores que influyen en el aprendizaje de la comprensión lectora y de la composición escrita: tres estudios realizados en aulas de Educación Infantil y Educación Primaria. Investigaciones sobre lectura, (3), 67-82. Doi: https://doi.org/10.37132/isl.v0i3.33

Martín-del-Campo, B. (1996). Automatización del acceso léxico: ¿̇es posible desde el lenguaje integrado? Cultura y Educación, 8(2), 103-113. https://doi. org/10.1174/113564096763277760

Martín-del-Campo, B. (2011). Enseñar a usar el lenguaje escrito a niños con necesidades educativas especiales. Revista de Logopedia, Foniatría y Audiología, 31(2), 82-95. doi:10.1016/S0214-4603(11)70176-0

Mataruna, G. y Garzón, C. (2015). La etnografía en el ámbito educativo: una alternativa metodológica de investigación al servicio docente. Educación y Desarrollo Social, 9(2), 192-205. doi:10.18359/reds.954 
Papanicolaou, A.; Simos, P.; Breier, J.; Fletcher, J.; Foorman, B.; Francis, D. y Davis, R. (2003). Brain mechanisms for reading in children with and without dyslexia: A review of studies of normal development and plasticity. Developmental Neuropsychology, 24(2-3), 593-612. doi:10.1080/87565641.2003.9651912

Pascual, M.R.; Madrid, D. y Estrada, L. (2018). Factores predominantes en el aprendizaje de la iniciación a la lectura. Revista Mexicana de Investigación Educativa, 79(23), 11211147. http://www.scielo.org.mx/pdf/rmie/v23n79/1405-6666-rmie-23-79-1121.pdf

Reglamento 2016/679 del Parlamento Europeo y del Consejo. Relativo a la protección de las personas físicas en lo que respecta al tratamiento de datos personales y a la libre circulación de estos datos y por el que se deroga la Directiva 95/46/CE.

Rivas, L. (2015). Metodología para el desarrollo de la comprensión lectora en el proceso enseñanza-aprendizaje. Dominio de las Ciencias, 1(1), 47-61. https:// dialnetuniriojaes.bibliotecauoc.idm.oclc.org/servlet/catart?codigo=5761664

Robinson, J. (2018). Evaluation of Teaching Methods to Improve Reading Performance of English Language Learners. Journal for the Advancement of Educational Research International, 12(1), 25-33. https://eric.ed.gov/?id=EJ1209451

Schreier, M. (2012). Qualitative content analysis in practice. Sage.

Share, D. L. (2008). On the Anglocentricities of current reading research and practice: the perils of overreliance on an" outlier" orthography. Psychological bulletin, 134(4), 584. https://doi.org/10.1037/0033-2909.134.4.584

Stanley, C.; Petscher, Y. y Catts, H. (2018). A longitudinal investigation of direct and indirect links between reading skills in kindergarten and reading comprehension in tenth grade. Read Writ, 31(1), 133-153. https://doi.org/10.1007/s11145-017-97776

Taylor, J.; Davis, M. y Rastle, K. (2017). Comparing and Validating Methods of Reading Instruction Using Behavioural and Neural Findings in an Artificial Orthography. Journal of Experimental Psychology, 146(6), 826-858. doi: 10.1037/xge0000301

Tighe, E.; Wagner, R. y Schatschneide, C. (2015). Applying a multiple group causal indicator modeling framework to the Reading comprehension skills of third, seventh, and tenth grade students. Reading and Writing, 28, 439-466. doi: 10.1007/s11145014-9532-1

Tobia, V. y Bonifacci, P. (2015). The simple view of reading in a transparent orthography: The stronger role of oral comprehension. Reading and Writing, 28(7), 939-957. https://doi.org/10.1007/s11145-015-9556-1 
Villar, D. y Vieiro, P. (2015). Métodos de lectura y acceso al léxico on-line en lectores principiantes. Ciencias Psicológicas, 9(2), 309-319. http://www.scielo.edu.uy/scielo. php?script=sci_arttext\&pid=S1688-42212015000300008\&lng=es\&tlng=es

Wang, Z.; Sabatini, J.; O’Reilly, T. y Weeks, J. (2019). Decoding and Reading Comprehension: A Test of the Decoding Threshold Hypothesis. Journal of Educational Psychology, 111(3), 387-401. doi: 10.1037/edu0000302

Yuste, C. y Yuste, D. (2011). BADyG: Batería de Aptitudes Diferenciales y Generales, Nivel E1, Renovado. Editorial CEPE (Ciencias de la Educación Preescolar \& Especial)

\footnotetext{
${ }^{1}$ Graduado en Maestro en Educación Infantil por la Universidad de Alicante (UA). 3er clasificado entre todos los trabajos del curso académico 2019-2020, por el trabajo final en el Máster Universitario de Dificultades del Aprendizaje y Trastornos del Lenguaje de la Universidad Oberta de Catalunya (UOC). Ejerciendo como Maestro en Educación Infantil desde el curso 2015-2016.

${ }^{2}$ Doctora en Psicología por la Universidad Nacional de Educación a Distancia (UNED). Profesora Titular en el Grado en Logopedia de la Universidad de Castilla-La Mancha (UCLM) y colaboradora docente en el Máster de Dificultades de Aprendizaje y Trastornos del Lenguaje de la UOC. Co-directora de la carrera Especialista en Dislexia y Discalculia de la UCLM. Investigadora principal del grupo de investigación INLOGO (Investigación en Logopedia). Su investigación se ha centrado en los campos del desarrollo y aprendizaje de la lengua escrita, vinculados de manera transversal con los temas de multiculturalidad, dificultades de aprendizaje e introducción de cambios pedagógicos en centros educativos, siempre relacionados con la lectura y la escritura. También diseña e implementa cursos para el profesorado universitario sobre la tutela de los Trabajos Fin de Grado y Fin de Máster, y sobre la enseñanza de la alfabetización académica.
} 